\title{
Protein binding pocket optimization for virtual high-throughput screening (vHTS) drug discovery
}

\section{Supporting Information}

Dimitris Gazgalis ${ }^{1}$, Mehreen Zaka ${ }^{1,2}$, Bilal Haider Abbasi ${ }^{2}$, Diomedes E. Logothetis ${ }^{1}$, Mihaly Mezei ${ }^{3}$, Meng Cui ${ }^{1 *}$

1Department of Pharmaceutical Sciences, Northeastern University School of Pharmacy, Boston, Massachusetts, 02115, USA

2Department of Biotechnology, Quaid-i-Azam University, Islamabad, Pakistan

${ }^{3}$ Department of Pharmacological Sciences, Icahn School of Medicine at Mount Sinai, New York, NY 10029, USA

${ }^{*}$ Correspondence should be addressed to M.C. (m.cui@northeastern.edu)

Running title: Binding pocket optimization by Monte Carlo 


\section{Supplemental 1}

Torsional angle sampling input

FILE Protein

TITL Protein for torsional angle sampling

TITL Solute molecules move, swap and torsions

HRDW VC32

! 32-bit vector

SVVC SPCC 13.00 ! Solvent cutoff

SUVC SPGC 13.0 ! MI on the solute

SUUC SPGC 17.0 ! Molec-based MI for intrasolute

PBCN HEXG 99.00 99.00 99.00 !hexagonal PBC

TEMP 998

NSLV 0 ! Only solute

STEP $0.00 \quad 00.0 \quad 0.55 \quad 40.010$

SVPT TIP3 TIP3 ! Solvent-solvent pot: CHARMM

SUPT CHRM

SETC DIEL 2.0

SLTA SMPL MMC FILE $(\mathrm{N}) \quad$ ! $(\mathrm{N})$ is the number of atoms

PRMF CHRM par_all22_prot_na.inp

!Torsion pot param file

PART UNIF RAND RAND 1.0

!Torsion strategy and frequency

TORD INPT (N) !Definiton of active torsions where $(\mathrm{N})$ is the number of torsions

$\ldots$ !Definitions of cative torsions from mcs file

CNFG RANI ASCI

!SWAP 0.2

TRAJ CHRM

!Specify swapping frequency

RUNS 10000001002000010005000

STOP SLFT 


\section{Supplemental 2}

Pocket size calculation input

FILE Protein

PRNT ECHO

HRDW VC32

! 32-bit vector

SVVC SPCC 9.05

! Solvent cutoff

SUVC MIGC 0.0

! MI on the solute

PBCN RECT 76.0 91.0 91.0 !Rectangular PBC

MOVE RAND

! Random selections

TEMP 298

NSLV 000

STEP $0.00 \quad 00.0 \quad 0.55 \quad 40.060$

! slt, slv stepsize, slt move freq.

SVPT TIP3 TIP3

SUPT CHRM

SAMP METC 0.5

MOLD $1(N) \quad$ IWhere $(N)$ is the number of atoms

PFRD CHRM toppar/par_all36_prot.prm

SLTA SMPL MMC FILE (N) !Where $(N)$ is the number of atoms GCEN CAVB RSIG ALTI
$1.0 \quad 2.5$
1000.0
2502502501000
110010000
50000 CNFG READ PDB

PRTG PDB ALLG AGLS 100.6

STOP 